\title{
Intestinal metastasis from primary ROSI-positive lung adenocarcinoma cancer patients responding to crizotinib
}

This article was published in the following Dove Press journal:

OncoTargets and Therapy

\author{
Hua-fei Chen ${ }^{1, *}$ \\ Qu-xia Zhang ${ }^{2, *}$ \\ You-cai Zhu' \\ Kai-qi Du' \\ Xiao-feng $\mathrm{Li}^{\prime}$ \\ Li-xin $\mathrm{Wu}^{\mathrm{l}}$ \\ Wen-xian Wang ${ }^{3}$ \\ Chun-wei $\mathrm{Xu}^{2}$ \\ 'Department of Thoracic Disease \\ Center, Zhejiang Rongjun Hospital, \\ Jiaxing, Zhejiang, China; ${ }^{2}$ Department \\ of Pathology, Fujian Cancer Hospital, \\ Fujian Medical University Cancer \\ Hospital, Fuzhou, Fujian, China; \\ ${ }^{3}$ Department of Chemotherapy, \\ Zhejiang Cancer Hospital, Hangzhou, \\ Zhejiang, China \\ *These authors contributed equally \\ to this work
}

\begin{abstract}
Small intestinal metastases from primary lung cancer are rare. Such patients have a poor prognosis. Early diagnosis of small intestinal metastases is difficult because of the low incidence of clinically apparent symptoms. The standard treatment for small intestinal metastases has not been established. A 69-year-old Chinese man presented for evaluation of a tumor in the right lower lung and mediastinal lymph node enlargement on clinical examination. The clinical stage was $\mathrm{cT}_{2} \mathrm{~N}_{2} \mathrm{M}_{0}$ (stage IIIA). Histologic examination of the tumor revealed lung adenocarcinoma. He could not tolerate surgery; hence, he received two chemotherapy regimens. However, the disease progressed. He had bloating after chemotherapy and decreased flatus. An abdominal CT scan showed an intestinal effusion with local intestinal obstruction. Medical treatment was ineffective; hence, he underwent a diagnostic laparoscopy. The pathologic evaluation suggested an intestinal metastatic adenocarcinoma from the primary lung cancer. Based on an real-time PCR assay, the tumor had a ROS1 fusion and responded well to crizotinib. The progression-free survival was 7 months. Physicians must be aware of the possibility of intestinal metastases from primary lung cancer. With an accurate diagnosis and thorough evaluation, patients may benefit from targeted therapy.
\end{abstract}

Keywords: lung adenocarcinoma, ROS1, crizotinib, metastasis, small intestine

\section{Introduction}

Among all neoplasms, lung cancer has the highest registered mortality rate worldwide. ${ }^{1,2}$ Despite advances in prevention, it has been reported that approximately $50 \%$ of lung cancer patients present with metastases at the time of diagnosis ${ }^{3}{ }^{3}$ however, as a result of advances in diagnosis and treatment, patient survival has increased in the last few years, which unfortunately facilitates the development of long-term metastases. ${ }^{4}$ Distant metastases from primary lung cancer are most commonly demonstrated in the contralateral lung, brain, liver, adrenal glands, and bones. ${ }^{5}$ Thus, gastrointestinal (GI) metastases are not common. Different studies have reported that GI metastases from primary lung cancer are infrequent, with an incidence ranging between $0.3 \%$ and $1.7 \%{ }^{6}$ Intestinal metastases may be asymptomatic and include obstruction, perforation, occult bleeding, and intussusception. Symptomatic intestinal metastases may be life-threatening and necessitate emergency surgery. Postmortem studies have estimated that the incidence of symptomatic metastatic lesions ranges from $4.6 \%$ to $14 \% .^{7}$ Thus, primary lung cancer metastasizing to the small bowel is actually not a rare occurrence.

Despite the increase in relevant publications, there is still no consensus on the management of patients with intestinal metastases from primary lung cancer, which 
generally occurs late in the course of the disease. According to some reports, the prognosis of patients with small intestinal metastases is very poor, with a survival time of days to a few months. ${ }^{8,9}$

Currently, lung adenocarcinoma is the most common subtype of non-small-cell lung cancer (NSCLC). ${ }^{10}$ With the development of gene detection, more and more driver gene alterations have been identified in patients with NSCLC, including epidermal growth factor receptor $(E G F R)$ and chromosomal rearrangements involving the anaplastic lymphoma kinase (ALK) gene, and the c-ros oncogene 1 (ROS1). Studies have shown that target drugs can improve the efficacy and survival of patients with gene mutations. ${ }^{11-14}$ Between $1 \%$ and $2 \%$ of patients with NSCLC harbor an ROS 1 rearrangement; the incidence is slightly higher in the East Asian population, with a frequency of $2 \%-3 \% .^{15,16}$ Crizotinib is a first-generation small molecule tyrosine kinase inhibitor which has been shown to be effective against lung cancers harboring ROS1 alterations. ${ }^{17}$ An objective response rate (ORR) of $72 \%$ and a median progression-free survival (PFS) of 19.2 months were reported in advanced ROS1-rearranged
NSCLC based on a Phase I crizotinib trial. ${ }^{11}$ Therefore, accurate diagnoses are important with respect to the treatment and prognosis of NSCLC patients. Indeed, patients may survive longer after appropriate treatment.

No study has reported intestinal metastases in a primary ROS1-positive lung cancer patient and the efficacy of crizotinib treatment. All protocols in the present study were approved by the Human Clinical and Research Ethics Committees of the Zhejiang Rongjun Hospital (Jiaxing, China). The patient provided written informed consent for the publication of case details and any accompanying images.

\section{Case report}

In March 2017, a 69-year-old Chinese man, who was a nonsmoker, presented to our hospital for the evaluation of a pulmonary mass. A computed tomography (CT) scan showed a mass in the right lower lung and mediastinal lymph node enlargement (Figure 1A). A brain-enhanced MRI and abdominal CT were unremarkable. Due to poor lung function, he could not tolerate surgery. He underwent bronchoscopy, and
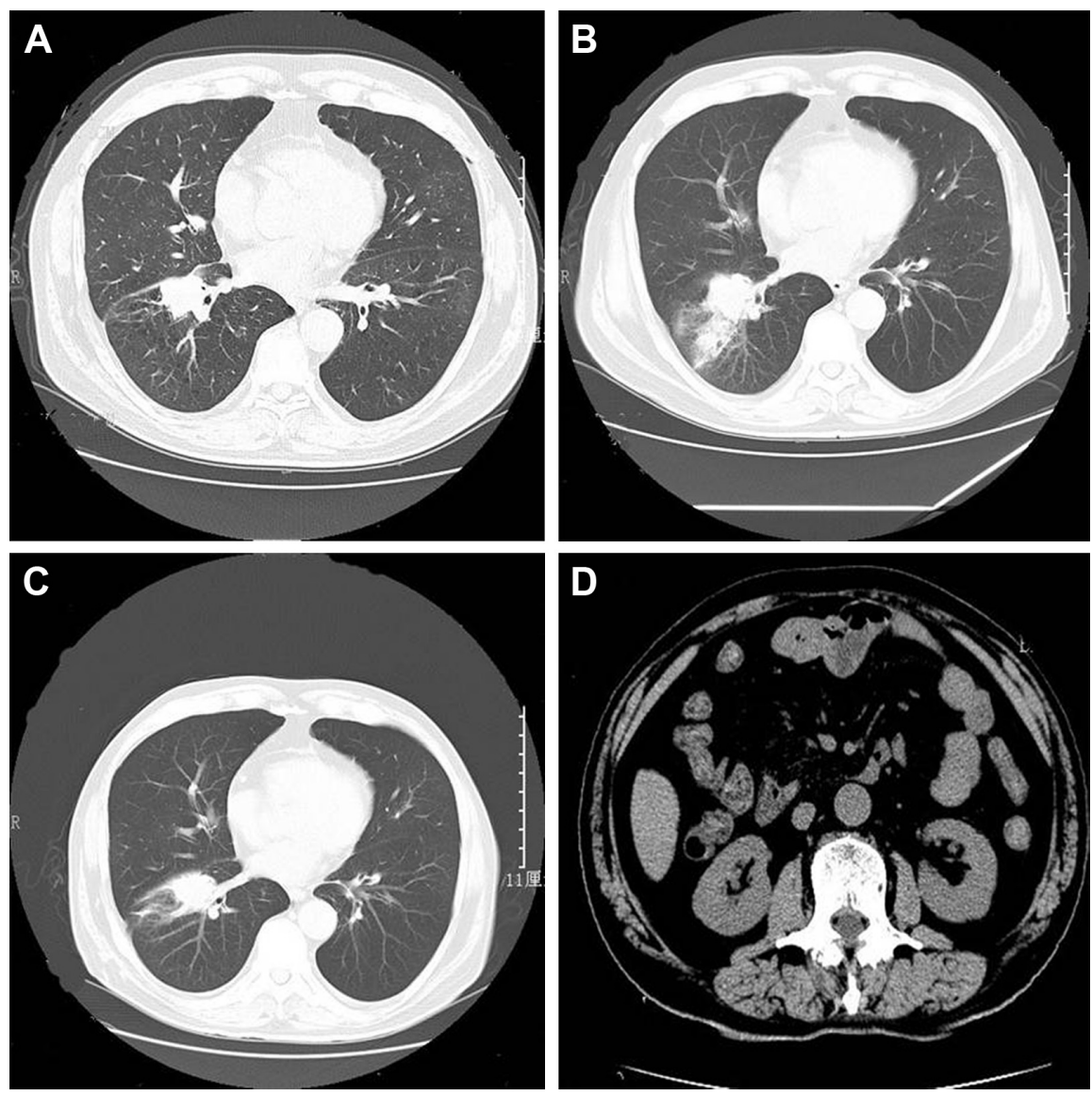

Figure I Computed tomography (CT) scans show (A) prior treatment of the lung tumor. (B) Cisplatin+cyclophosphamide regimen after chemotherapy. (C) Pemetrexed combined with cisplatin regimen after chemotherapy. (D) Intestinal obstruction after pemetrexed combined with cisplatin regimen. 

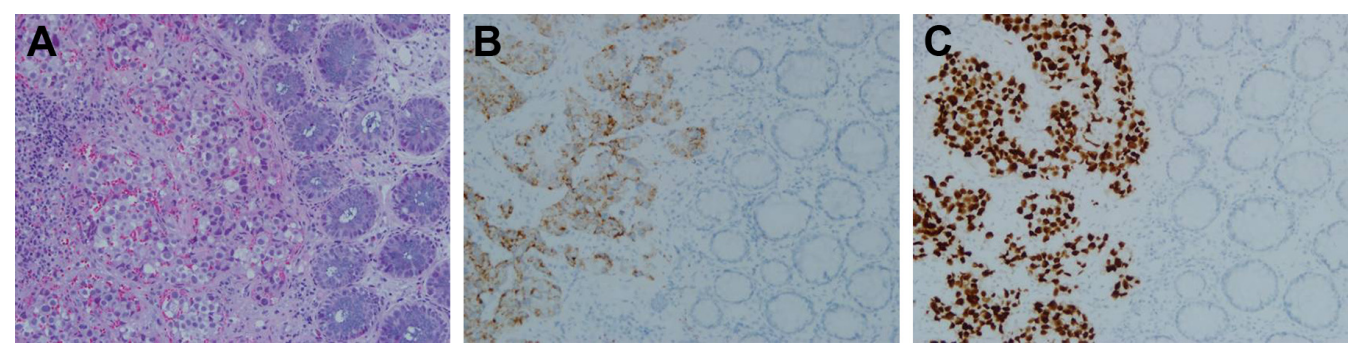

Figure $2(\mathbf{A})$ Hematoxylin and eosin $(\mathrm{H} \& \mathrm{E})$ staining revealed adenocarcinoma $(\mathrm{H} \& \mathrm{E} \times 100)$. Immunohistochemical (IHC) analysis revealed that the lung tumor cells were positive for Napsin A (B) and TTF-I (C) $(\mathrm{H} \& \mathrm{E} \times \mathrm{I00})$.

immunohistochemical staining was positive for the expression of TTF- 1 and Napsin A and negative for CK5/6 and P40 (Figure 2). Tumor tissue was shown to have wild-type EGFR variants by ARMS (AmoyDx, Xiamen, China) and ALK protein expression was negative based on the Ventana ALK IHC assay (Ventana Medical Systems; Hoffman-La Roche Ltd., Basel, Switzerland). The clinical stage was $\mathrm{cT}_{2} \mathrm{~N}_{2} \mathrm{M}_{0}$ (stage IIIA) according to the seventh edition of TNM staging. He underwent two cycles of chemotherapy (docetaxel, $75 \mathrm{mg} / \mathrm{m}^{2}$ on days 1 and 8 ; and carboplatin area under curve $=5$ on day 1 [DP]). The disease progressed after two cycles of DP chemotherapy (Figure 1B), thus the chemotherapy regimen was changed to pemetrexed $\left(500 \mathrm{mg} / \mathrm{m}^{2}\right.$ on day 1$)$, combined with cisplatin (25 $\mathrm{mg} / \mathrm{m}^{2}$ on days $1-3$ ) plus bevacizumab (5 mg/ $\mathrm{kg}$ on day 1). Lung lesion control was stable (Figure 1C); however, after three cycles the patient had bloating and decreased flatus, and an abdominal CT scan showed an intestinal effusion with local intestinal obstruction (Figures $1 \mathrm{D}$ and $3 \mathrm{~A}$ ). Because medical treatment was ineffective, he underwent a diagnostic laparoscopy. The pathologic examination suggested an intestinal metastatic adenocarcinoma from a primary lung cancer. Using an real-time PCR (RT-PCR) assay (ACCB Biotech, Beijing, China), we found that the tumor had an ROS1 fusion (Figure 4). The patient started crizotinib therapy in September 2017 and had a good response. The efficacy was considered to be a partial response (PR) according to the Response Evaluation Criteria 1.1 in Solid Tumors (Figure 3B). During crizotinib therapy, he had grade 1 GI reactions. There were no other treatment-related adverse events, including liver dysfunction, rashes, renal function, or cordis damage. The disease progressed after 7 months (Figure 3C), and other treatment was initiated. He is alive at this writing with an overall survival $>14$ months.

\section{Discussion}

This is the first report of a patient with an ROS1 fusion and intestinal metastases from a primary lung cancer who responded to crizotinib treatment. Considering this rare condition and the good response to target therapy, we encourage clinicians to be aware of the existence of intestinal metastases from primary lung cancer in NSCLC patients. Upon diagnosis, the appropriate treatment must be urgently initiated.

Small intestinal metastases from lung cancer are actually not rare. Patients with a history of lung cancer will present with different symptoms. The majority of patients with intestinal metastases from primary lung are asymptomatic. ${ }^{6}$ This finding has led to a discrepancy between the estimated incidence in clinical and postmortem studies. ${ }^{18}$ This clinical underdiagnosis likely occurs because the intestinal symptoms experienced by the patient overlap with the adverse effects of chemotherapy. ${ }^{19}$ The most frequently observed symptoms without associated complications are abdominal
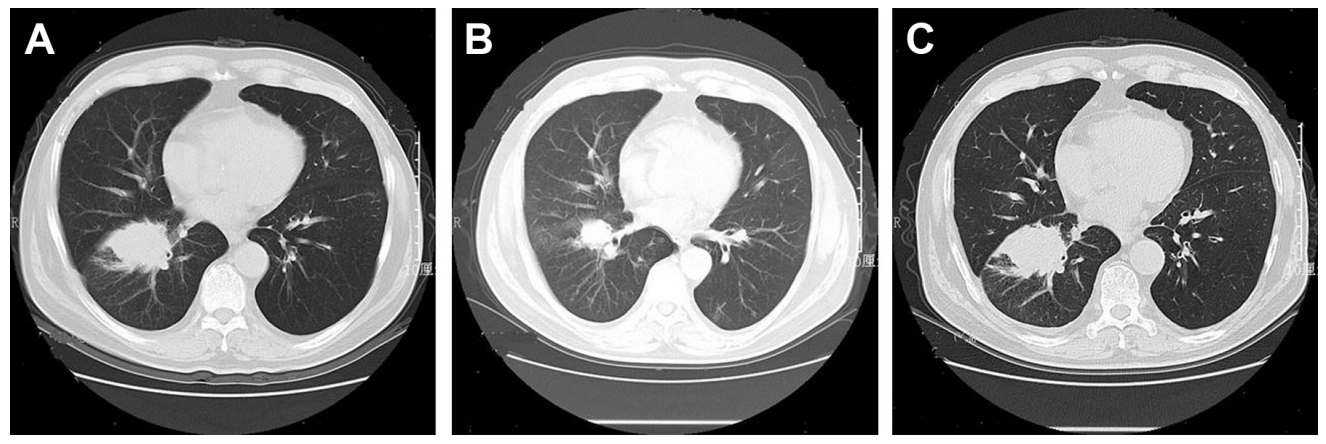

Figure 3 Computed tomography (CT) scan shows (A) postoperative intestinal obstruction and crizotinib before treatment. (B) Two months after crizotinib treatment. (C) Disease progression after 7 months of crizotinib treatment. 


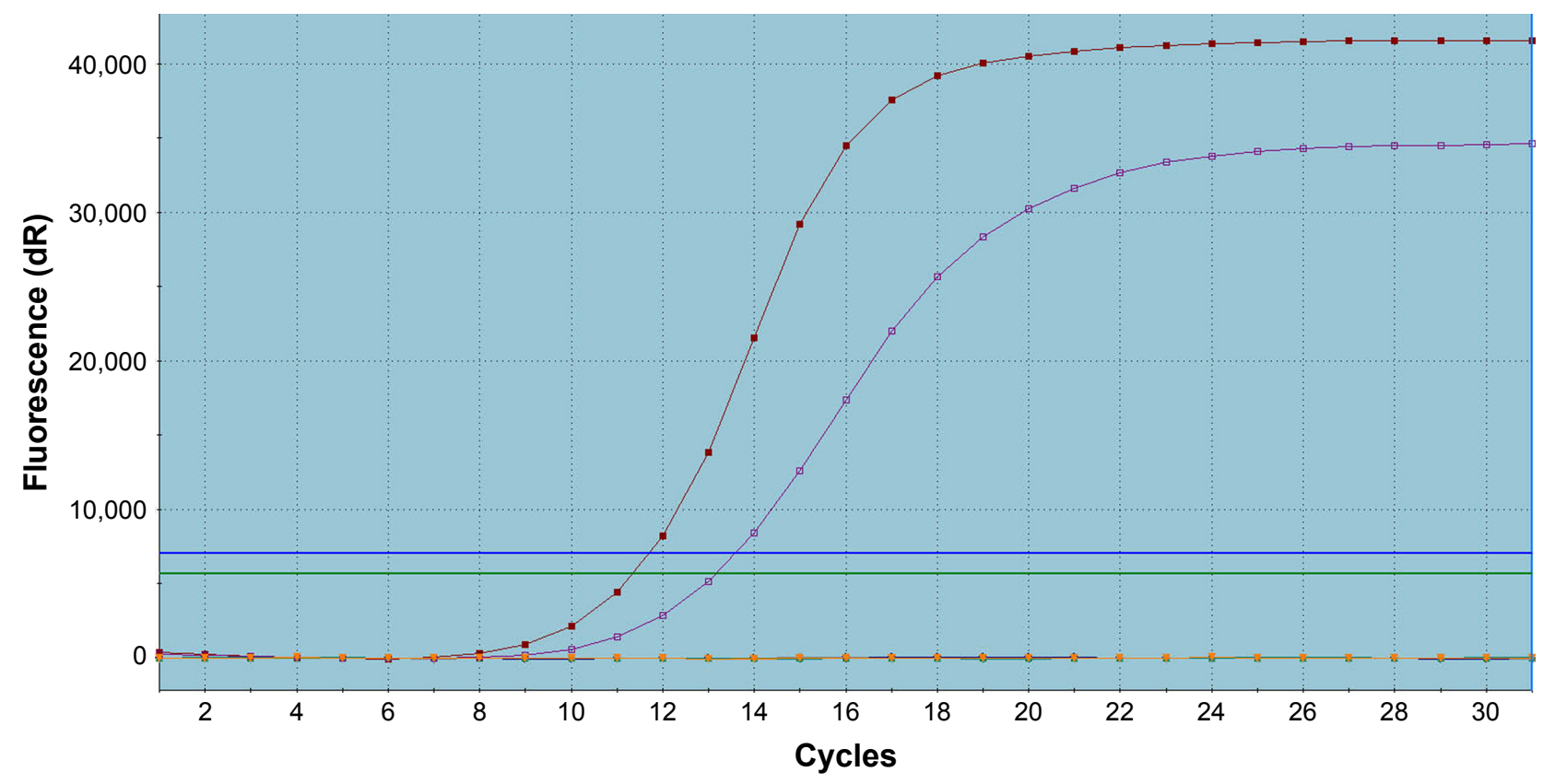

Figure 4 Schema shows tumor with drivers of ROSI gene positive by RT-PCR. Purple, brown, and orange represent the sample, positive control, and negative control, respectively.

Abbreviation: RT-PCR, real-time PCR.

pain ( $50 \%$ of patients) and weight loss. ${ }^{9,20}$ Among the most frequent complications, small bowel perforation has been described, as well as the intestinal obstruction that can occur as a result of an occlusive mass or by invagination of the affected segment of the liver. ${ }^{18,21}$ Finally, an acute digestive hemorrhage has been described. In the present case, obstruction of the small intestine represents the most frequently observed complication (28.6\%), reaching up to $35 \%$ according to Di et al. ${ }^{9}$ In addition, Ying et al ${ }^{22}$ reported a case with metastasis to the small intestine from a primary lung cancer in a patient who presented with anemia and melena. In our report, the patient had bloating and decreased flatus after chemotherapy, which indicated obstruction of the small intestine and the need to identify adverse reactions to chemotherapy. Therefore, in the course of advanced lung cancer, we should realize that every symptom, including abdominal pain, reduced flatus, anemia, melena, and hemorrhage, is present in such patients.

Based on our review of the literature, the prognosis of a patient with small intestinal metastases from primary lung cancer is generally very poor. ${ }^{8}$ The average survival ranges between 1 and 6 months..$^{3,9,19,23}$ The largest series published in the literature reports a retrospective analysis of 100 previously published cases, including autopsies, that showed a median survival of 2.3 months and concluded that the main risk factors associated with mortality were as follows: age $>70$ years, intestinal perforation, and presence of extraintestinal metastases. ${ }^{9}$ The other review demonstrated that the median overall survival of 91 GI metastases from primary lung cancer patients was 4 months (95\% CI: 2.68-5.31). ${ }^{24}$ Despite the published data, there is still no standardized treatment, and none of the treatments described appear to have a significant impact on survival. In the case of perforation, intestinal obstruction, or massive hemorrhage, surgical treatment is usually the most rational approach; however, surgical treatment should not prolong patient survival. Selected patients, however, may survive longer after appropriate treatment.

In our study, a very poor response to chemotherapy was observed using docetaxel or pemetrexed with platinum. In the case reported by Ying et al, ${ }^{22}$ the patient with $M E T$ amplification experienced disease progression during chemotherapy or crizotinib treatment. Fortunately, our patient with an ROS1 fusion benefited from crizotinib treatment. Crizotinib, an ALK/ROS1/MET inhibitor, has a reported ORR of $71.7 \%$ and a median PFS of 15.9 months in East Asian patients with ROS1-positive advanced NSCLC (127 patients). ${ }^{25} \mathrm{Li}$ et a ${ }^{26}$ analyzed the efficacy of crizotinib in 36 patients with different types of ROS1 fusion partners and indicated ORR of $83.3 \%$ in all patients, whereas the ORR was $94.11 \%$ and $73.68 \%$ in the non-CD74-ROS1 and CD74-ROS1 groups, respectively. As compared to the CD74-ROS1 group, the non-CD74-ROS1 group had a significantly longer PFS (17.63 vs 12.63 months; $P=0.048)$. In the present case, the efficacy was a PR and the PFS was 7 months. Despite the benefits from crizotinib treatment, the PFS was shorter than reported. 
Based on the RT-PCR detection assay, we did not ascertain the specific subtype of ROS1 fusion, which can be verified by next-generation sequencing.

We should be aware of the phenomenon of intestinal metastases from primary lung cancer. The difficulty in detection, however, makes the development of prospective studies more challenging. Therefore, we should be aware of the symptoms on the diagnosis and choose appropriate therapy to improve patient prognosis.

\section{Acknowledgments}

This study was supported in part by grants from the Science and Technology Planning Project of Zhejiang Province (2015C33194), the Medical Scientific Research Foundation of Zhejiang Province of China (2019RC027), the Technology Bureau of Jiaxing City of Zhejiang Province (2016AY23087, 2017BY18050), and the National Clinical Key Specialty Construction Program (2013).

\section{Disclosure}

The authors report no conflicts of interest in this work.

\section{References}

1. Siegel RL, Miller KD, Jemal A. Cancer statistics, 2018. CA Cancer J Clin. 2018;68(1):7-30.

2. Chen W, Zheng R, Baade PD, et al. Cancer statistics in China, 2015. CA Cancer J Clin. 2016;66(2):115-132.

3. Goh BK, Yeo AW, Koong HN, Ooi LL, Wong WK. Laparotomy for acute complications of gastrointestinal metastases from lung cancer: is it a worthwhile or futile effort? Surg Today. 2007;37(5):370-374.

4. Malik PS, Raina V. Lung cancer: prevalent trends \& emerging concepts. Indian J Med Res. 2015;141(1):5-7.

5. Lardinois D, Weder W, Hany TF, et al. Staging of non-small-cell lung cancer with integrated positron-emission tomography and computed tomography. N Engl J Med. 2003;348(25):2500-2507.

6. Jevremovic V. Is Gastrointestinal Metastasis of Primary Lung Malignancy as Rare as Reported in the Literature? A Comparison Between Clinical Cases and Post-mortem Studies. Oncol Hematol Rev. 2016;12(01):51-57.

7. Yoshimoto A, Kasahara K, Kawashima A. Gastrointestinal metastases from primary lung cancer. Eur J Cancer. 2006;42(18):3157-3160.

8. Kobayashi N, Watanabe S, Kubota K. Small intestinal metastasis from carcinoma of the lung. Clin Gastroenterol Hepatol. 2011;9(10):e103.

9. di JZ, Peng JY, Wang ZG. Prevalence, clinicopathological characteristics, treatment, and prognosis of intestinal metastasis of primary lung cancer: a comprehensive review. Surg Oncol. 2014;23(2):72-80.
10. Stinchcombe TE, Socinski MA. Current treatments for advanced stage non-small cell lung cancer. Proc Am Thorac Soc. 2009;6(2): 233-241.

11. Shaw AT, Ou SH, Bang YJ, et al. Crizotinib in ROS1-rearranged nonsmall-cell lung cancer. $N$ Engl J Med. 2014;371(21):1963-1971.

12. Maemondo M, Inoue A, Kobayashi K, et al. Gefitinib or chemotherapy for non-small-cell lung cancer with mutated EGFR. $N$ Engl $J$ Med. 2010;362(25):2380-2388.

13. Rosell R, Carcereny E, Gervais R, et al. Erlotinib versus standard chemotherapy as first-line treatment for European patients with advanced EGFR mutation-positive non-small-cell lung cancer (EURTAC): a multicentre, open-label, randomised phase 3 trial. Lancet Oncol. 2012;13(3): 239-246.

14. Shaw AT, Yeap BY, Solomon BJ, et al. Effect of crizotinib on overall survival in patients with advanced non-small-cell lung cancer harbouring ALK gene rearrangement: a retrospective analysis. Lancet Oncol. 2011;12(11):1004-1012.

15. Davies KD, Le AT, Theodoro MF, et al. Identifying and targeting ROS1 gene fusions in non-small cell lung cancer. Clin Cancer Res. 2012; 18(17):4570-4579.

16. Kohno T, Nakaoku T, Tsuta K, et al. Beyond ALK-RET, ROS1 and other oncogene fusions in lung cancer. Transl Lung Cancer Res. 2015; $4(2): 156-164$.

17. Yasuda H, de Figueiredo-Pontes LL, Kobayashi S, Costa DB. Preclinical rationale for use of the clinically available multitargeted tyrosine kinase inhibitor crizotinib in ROS1-translocated lung cancer. J Thorac Oncol. 2012;7(7):1086-1090.

18. Kim MS, Kook EH, Ahn SH, et al. Gastrointestinal metastasis of lung cancer with special emphasis on a long-term survivor after operation. J Cancer Res Clin Oncol. 2009;135(2):297-301.

19. Gonzalez-Tallon AI, Vasquez-Guerrero J, Garcia-Mayor MA. Colonic metastases from lung carcinoma: A case report and review of the literature. Gastroenterology Res. 2013;6(1):29-33.

20. Misra SP, Dwivedi M, Misra V, Dharmani S, Gupta M. Duodenal metastases from squamous cell carcinoma of the lung: endoscopic management of bleeding and biliary and duodenal obstruction. Indian J Gastroenterol. 2004;23(5):185-186.

21. Kini S, Kapadia RM, Amarapurkar A. Intussusception due to intestinal metastasis from lung cancer. Indian J Pathol Microbiol. 2010;53(1): 141-143.

22. Ying X, Wang M, Verma V, et al. Metastatic spread of solid subtype lung adenocarcinoma to the small intestine with anemia and melena: A case report. Medicine. 2017;96(34):e7768.

23. Lee P-C, Lo C, Lin MT, Liang JT, Lin BR. Role of surgical intervention in managing gastrointestinal metastases from lung cancer. World J Gastroenterol. 2011;17(38):4314-4320.

24. Balla A, Subiela JD, Bollo J, et al. Gastrointestinal metastasis from primary lung cancer. Case series and systematic literature review. Cir Esp. 2018;96(4):184-197.

25. Wu YL, Yang JC, Kim DW, et al. Phase II Study of Crizotinib in East Asian Patients With ROS1-Positive Advanced Non-Small-Cell Lung Cancer. J Clin Oncol. 2018;36(14):JCO2017755587.

26. Li Z, Shen L, Ding D, et al. Efficacy of Crizotinib among Different Types of ROS1 Fusion Partners in Patients with ROS1-Rearranged Non-Small Cell Lung Cancer. J Thorac Oncol. 2018;13(7):987-995.
OncoTargets and Therapy

\section{Publish your work in this journal}

OncoTargets and Therapy is an international, peer-reviewed, open access journal focusing on the pathological basis of all cancers, potential targets for therapy and treatment protocols employed to improve the management of cancer patients. The journal also focuses on the impact of management programs and new therapeutic agents and protocols on

\section{Dovepress}

patient perspectives such as quality of life, adherence and satisfaction. The manuscript management system is completely online and includes a very quick and fair peer-review system, which is all easy to use. Visit http://www.dovepress.com/testimonials.php to read real quotes from published authors. 